KODAI MATH. SEM. REP.

21 (1969), 167-174

\title{
GENERALIZATIONS OF THE CONNECTION OF TZITZÉICA
}

\author{
By Kentaro Yano
}

Dobrescu [1] has recently studied what he calls the connection of Tzitzéica [4] on hypersurfaces in a Euclidean space.

The main purpose of the present paper is to define the connection of Tzitzéica on hypersurfaces in an affinely connected manifold along which a torse-forming [6] or a concurrent vector field [5] is given and to study the properties of the connection of Tzitzéica thus defined.

\section{$\S 1$. The connection of Tzitzéica on a hypersurface in a centro-affine space.}

Let $A^{n}$ be an $n$-dimensional centro-affine space ( $\left.n \geqq 3\right)$, that is, an affine space in which a point $\mathrm{O}$ is specified. Then any point $\mathrm{P}$ in $A^{n}$ is represented by the socalled position vector $X=\overrightarrow{\mathrm{OP}}$. This means that with every point $\mathrm{P}$ of $A^{n}$, there is associated a vector $X$.

We now assume that there is given a hypersurface $V^{n-1}$ in $A^{n}$ and denote by

$$
X=X\left(u^{1}, u^{2}, \cdots, u^{n-1}\right)
$$

the parametric representation of $V^{n-1}$, where $\left(u^{a}\right)(a, b, c, \cdots=1,2,3, \cdots, n-1)$ are local parameters on $V^{n-1}$ such that vectors

$$
X_{b}=\partial_{b} X
$$

tangent to $V^{n-1}$ are linearly independent, $\partial_{b}$ denoting the differential operators $\partial_{b}=\partial / \partial u^{b}$.

We assume that the vector $X$ at $\mathrm{P}$ on $V^{n-1}$ is never tangent to $V^{n-1}$, that is, the vector $X$ is linearly independent of $X_{b}$.

We then have, for the vectors $\partial_{c} X_{b}$, the equations of the form

$$
\partial_{c} X_{b}=\Gamma_{c b}^{a} X_{a}+h_{c b} X,
$$

where $\Gamma_{c}^{a} b$, symmetric in $c$ and $b$, define an affine connection on $V^{n-1}$ called the connection of Tzitéica [1], [4] and $h_{c b}$, symmetric in $c$ and $b$, define a tensor field on $V^{n-1}$ called the second fundamental tensor.

The equations (1.1) are so-called equations of Gauss for the $V^{n-1}$, the pseudo-

Received September 5, 1968. 
affine normal being the vector $X$ associated with the point $\mathrm{P}$ on $V^{n-1}$. The equations of Weingarten in this case are

$$
\partial_{c} X=X_{c} .
$$

Computing the integrability conditions of (1.1) and (1.2) regarded as a completely integrable system of partial differential equations with unknown vectors $X_{b}$ and $X$, we find

$$
R_{d c b}{ }^{a}+\delta_{d}^{a} h_{c b}-\delta_{c}^{a} h_{d b}=0,
$$

and

$$
\nabla_{d} h_{c b}-\nabla_{c} h_{d b}=0
$$

where

$$
R_{d c b}^{a}=\partial_{d} \Gamma_{c b b}^{a}-\partial_{c} \Gamma_{d b}^{a}+\Gamma_{d}^{a}{ }_{e}^{a} \Gamma_{c b}^{e}-\Gamma_{c}{ }^{a}{ }_{e} \Gamma_{d b}{ }^{e}
$$

are components of the curvature tensor of the connection of Tzitzéica and $\nabla_{d}$ denotes the covariant differentiation with respect to the connection of Tzitzéica.

From (1.3) we have

$$
R_{c b}+(n-2) h_{c b}=0,
$$

where $R_{c b}$ are components of the Ricci tensor

$$
R_{c b}=R_{a c b}{ }^{a} \text {. }
$$

Equation (1.6) shows that the Ricci tensor of the connection of Tzitzéica is symmetric:

$$
R_{c b}=R_{b c},
$$

from which we see that the connection of Tzitzéica is volume-preserving.

From (1. 3), (1.4) and (1.6), we find, for $n \geqq 3$,

$$
R_{d c b}^{a}-\frac{1}{n-2}\left(\delta_{d}^{a} R_{c b}-\delta_{c}^{a} R_{d b}\right)=0
$$

and

$$
\nabla_{d} R_{c b}-\nabla_{c} R_{d b}=0,
$$

which show that the connection of Tzitzéica is projectively flat.

Conversely suppose that there is given, in an $(n-1)$-dimensional differentiable manifold $V^{n-1}(n \geqq 3)$, a symmetric affine connection $\Gamma_{c b}^{a}$ which is volume-preserving and projectively flat. We then have equations (1.8), (1.9) and (1.10), which show that the system of partial differential equations 
(1. 11)

$$
\partial_{c} X_{b}=\Gamma_{c b}^{a} X_{a}+\frac{1}{n-2} R_{c b} X,
$$

$$
\partial_{c} X=X_{c}
$$

is completely integrable. Thus we have

Proposition 1.1. A symmetric volume-preserving projectively fiat affine connection is always realized as a connection of Tzitzéica on a hypersurface in a centro-affine space.

\section{§ 2. A characterization of projectively flat manifold.}

Let $M^{n}$ be an $n$-dimensional differentiable manifold $(n \geqq 3)$ with a symmetric affine connection $V$ with components $\Gamma_{j}{ }^{h} i(x)$, where $\left(x^{h}\right)$ are local coordinate system in $M^{n}$ and the indices $h, i, j, \cdots$ run over the range $\{1,2,3, \cdots, n\}$.

The curvature tensor field $R$ of $M^{n}$ is given by

$$
R(Z, Y) X=\nabla_{Z} \nabla_{Y} X-\nabla_{Y} \nabla_{Z} X-\nabla_{[Z, Y]} X
$$

for any vector fields $Z, Y, X$ in $M^{n}$.

We assume that the vector $R(Z, Y) X$ is always in the three-dimensional linear space spanned by the vectors $Z, Y$ and $X$, that is,

$$
R(Z, Y) X=\gamma(Y, X) Z+\beta(Z, X) Y+\alpha(Z, Y) X,
$$

where $\gamma, \beta$ and $\alpha$ are all tensors of type $(0,2)$.

Since $R(Z, Y) X$ is skew-symmetric in $Z$ and $Y$, we see that

$$
\begin{gathered}
\gamma(Y, X)+\beta(Y, X)=0, \\
\alpha(Z, Y)+\alpha(Y, Z)=0
\end{gathered}
$$

and consequently we have

$$
R(Z, Y) X=\gamma(Y, X) Z-\gamma(Z, X) Y+\alpha(Z, Y) X
$$

$\alpha(Z, Y)$ being skew-symmetric.

If $(\mathrm{P})$ is satisfied, then we say that the affine connection has the property $(\mathrm{P})$.

Denoting the local components of $R, \gamma$ and $\alpha$ by $R_{k j i}{ }^{h},-\gamma_{j i}$ and $\alpha_{j i}$ respectively, we find, from $(\mathrm{P})$,

$$
R_{k j i}{ }^{h}+\delta_{k}^{h} \gamma_{j i}-\delta_{j}^{h} \gamma_{k i}-\alpha_{k j} \delta_{i}^{h}=0,
$$

from which, by contraction with respect to $k$ and $h$,

$$
R_{j i}+(n-1) \gamma_{j i}-\alpha_{i \jmath}=0,
$$

and, by contraction with respect to $i$ and $h$, 


$$
-R_{k j}+R_{j k}+\gamma_{j k}-\gamma_{k j}-n \alpha_{k j}=0,
$$

or

$$
-R_{\imath j}+R_{j i}+\gamma_{j i}-\gamma_{\imath j}-n \alpha_{i j}=0
$$

by virtue of the first Bianchi identity

$$
R_{k j i}^{h}+R_{j i k^{h}}+R_{i k j}{ }^{h}=0 .
$$

Forming (2.2) $\times n-(2.3)$, we find

$$
(n-1) R_{j i}+R_{\imath j}+\left(n^{2}-n-1\right) \gamma_{j i}+\gamma_{\imath j}=0,
$$

from which

$$
(n-1) R_{i j}+R_{j i}+\left(n^{2}-n-1\right) \gamma_{i j}+\gamma_{j i}=0 .
$$

Forming (2.4) $\times\left(n^{2}-n-1\right)-(2.5)$, we find

$$
\left[(n-1)\left(n^{2}-n-1\right)-1\right] R_{j i}+\left[\left(n^{2}-n-1\right)-(n-1)\right] R_{\imath j}+\left[\left(n^{2}-n-1\right)^{2}-1\right] \gamma_{j i}=0,
$$

or

(2.6)

$$
n R_{j i}+R_{\imath j}+\left(n^{2}-1\right) \gamma_{j i}=0
$$

from which

$$
\gamma_{j i}=-\frac{1}{n^{2}-1}\left(n R_{j i}+R_{\imath j}\right)
$$

and

$$
\gamma_{j i}-\gamma_{i j}=-\frac{1}{n+1}\left(R_{j i}-R_{\imath j}\right)
$$

Substituting (2.8) into (2.3), we find

$$
\alpha_{j i}=-\frac{1}{n+1}\left(R_{j i}-R_{\imath j}\right)
$$

that is,

$$
\alpha_{j i}=\gamma_{j i}-\gamma_{\imath \jmath}
$$

Thus (2.1) gives

$$
R_{k j i}{ }^{h}+\delta_{k}^{h} \gamma_{j i}-\delta_{j}^{h} \gamma_{k i}-\left(\gamma_{k j}-\gamma_{j k}\right) \delta_{i}^{h}=0,
$$

where $\gamma_{j i}$ is given by (2.7), and consequently the manifold $M^{n}(n \geqq 3)$ is projectively flat. Thus we have 
PROPOSITION 2.1. When a symmetric affine connection in an $n(\geqq 3)$-dimensional manifold has property (P), the manifold is projectively flat. (See, Ogiue [2])

PROPOSITION 2.2. When the curvature tensor of a symmetric affine connection in an $n(\geqq 3)$-dimensional manifold satisfies an equation of the form (2.1), the manifold is projectively flat.

$\S 3$. Fundamental equations of a hypersurface in a manifold with symmetric affine connection.

We next consider a hypersurface $V^{n-1}$ in $M^{n}$ and let

$$
x^{h}=x^{h}\left(u^{a}\right)
$$

be its parametric representation. The rank of

$$
B_{b}{ }^{h}=\partial_{b} x^{h}
$$

is assumed to be $n-1$ everywhere along $V^{n-1}$.

We take a vector field $C^{h}$ defined along $V^{n-1}$ such that $C^{h}$ is linearly independent of $B_{b}{ }^{h}$ and consequently the vectors $B_{b}{ }^{h}$ and $C^{h}$ form a frame on $V^{n-1}$. We denote by $B^{a}{ }_{\imath}$ and $C_{\imath}$ the components of covectors which form the dual coframe.

If we put

$$
\Gamma_{c b}^{a}=\left(\partial_{c} B_{b}{ }^{h}+\Gamma_{j}{ }_{i}{ }_{i} B_{c}{ }^{j} B_{b}{ }^{i}\right) B^{a}{ }_{h},
$$

then $\Gamma_{c}{ }^{a}{ }_{b}$ define a symmetric affine connection induced on the hypersurface with respect to the pseudo-affine normal $C^{h}$.

Then the so-called van der Waerden-Bortolotti covariant derivative of $B_{b}{ }^{h}$ :

$$
\nabla_{c} B_{b}{ }^{h}=\partial_{c} B_{b}{ }^{h}+\Gamma_{j}^{h}{ }_{i} B_{c}{ }^{j} B_{b}{ }^{2}-\Gamma_{c}{ }^{a}{ }_{b} B_{a}{ }^{h}
$$

is written as

$$
\nabla_{c} B_{b}{ }^{h}=h_{c b} C^{h},
$$

where $h_{c b}$ is the second fundamental tensor of the hypersurface $V^{n-1}$ with respect to $C^{h}$. The equation (3.4) is that of Gauss of the hypersurface $V^{n-1}$. The equation of Weingarten of the hypersurface $V^{n-1}$ is given by

$$
\nabla_{c} C^{h}=-h_{c}{ }^{a} B_{a}{ }^{h}+l_{c} C^{h},
$$

where $h_{c}{ }^{a}$ are components of the second fundamental tensor and $l_{c}$ those of the third fundamental tensor of $V^{n-1}$.

From (3.4) and (3.5), we find

$$
\begin{aligned}
& R_{k j i}{ }^{h} B_{d}{ }^{k} B_{c}{ }^{j} B_{b}{ }^{i} \\
= & {\left[K_{d c b}{ }^{a}-\left(h_{d}{ }^{a} h_{c b}-h_{c}{ }^{a} h_{d b}\right)\right] B_{a}{ }^{h}+\left[\nabla_{d} h_{c b}-\nabla_{c} h_{d b}+l_{d} h_{c b}-l_{c} h_{d b}\right] C^{h} }
\end{aligned}
$$


and

$$
\begin{aligned}
& R_{k j i}{ }^{h} B_{d}{ }^{k} B_{c}{ }^{\jmath} C^{\imath} \\
= & -\left[\nabla_{d} \nabla_{c}^{a}-\nabla_{c} h_{d}{ }^{a}-l_{d} h_{c}{ }^{a}+l_{c} h_{d}^{a}\right] B_{a}{ }^{h}+\left[\nabla_{d} l_{c}-\nabla_{c} l_{d}-h_{d a} h_{c}{ }^{a}+h_{c a} h_{d}^{a}\right] C^{h}
\end{aligned}
$$

by virtue of the Ricci identities

$$
\nabla_{d} \nabla_{c} B_{b}{ }^{h}-\nabla_{c} \nabla_{d} B_{b}{ }^{h}=R_{k j i}{ }^{h} B_{d}{ }^{k} B_{c}{ }^{j} B_{b}{ }^{2}-K_{d c b}{ }^{a} B_{a}{ }^{h}
$$

and

$$
\nabla_{d} \nabla_{c} C^{h}-\nabla_{c} \nabla_{d} C^{h}=R_{k j i}^{h} B_{d}^{k} B_{c}{ }^{\jmath} C^{\imath} .
$$

We now assume that the hypersurface $V^{n-1}$ has the property that

$$
R\left(Z^{\prime}, Y^{\prime}\right) X^{\prime}=\gamma\left(Y^{\prime}, X^{\prime}\right) Z^{\prime}-\gamma\left(Z^{\prime}, X^{\prime}\right) Y^{\prime}+\alpha\left(Z^{\prime}, Y^{\prime}\right) X^{\prime}
$$

is satisfied for any vector fields $Z^{\prime}, Y^{\prime}, X^{\prime}$ tangent to the hypersurface. Denoting the local components of $\gamma$ and $\alpha$ on the hypersurface by $-\gamma_{c b}$ and $\alpha_{c b}$ respectively, we find

$$
R_{k j i}{ }^{h} B_{d}^{k} B_{c}^{j} B_{b}{ }^{2}+\left(\delta_{d}^{a} \gamma_{c b}-\delta_{c}^{a} \gamma_{d b}-\alpha_{d c} \delta_{b}^{a}\right) B_{a}^{h}=0 .
$$

Substituting (3.8) into (3.6), we find

$$
\left[K_{d c b}{ }^{a}+\delta_{d}^{a} \gamma_{c b}-\delta_{c}^{a} \gamma_{d b}-\alpha_{d c} \delta_{b}^{a}-\left(h_{d}{ }^{a} h_{c b}-h_{c}{ }^{a} h_{d b}\right)\right] B_{a}{ }^{h}+\left[\nabla_{d} h_{c b}-\nabla_{c} h_{d b}+l_{d} h_{c b}-l_{c} h_{d b}\right] C^{h}=0,
$$

from which

$$
K_{d c b}{ }^{a}+\delta_{d}^{a} \gamma_{c b}-\delta_{c}^{a} \gamma_{d b}-\alpha_{d c} \delta_{b}^{a}-\left(h_{d}{ }^{a} h_{c b}-h_{c}{ }^{a} h_{d b}\right)=0
$$

and

$$
\nabla_{d} h_{c b}-\nabla_{c} h_{d b}+l_{d} h_{c b}-l_{c} h_{d b}=0 .
$$

\section{§4. Generalizations of the connection of Tzitzéica.}

Suppose that a vector field $C^{h}(x)$ is given in $M^{n}$ and satisfies

$$
\nabla_{i} C^{h}=\alpha \delta_{i}^{h}+\beta_{i} C^{h},
$$

where $\alpha$ is a scalar field and $\beta_{i}$ a covector field in $M^{n}$.

We then say that the vector field $C^{h}$ is torse-forming because if we develop the vetor field $C^{h}$ along a curve in the manifold $M^{n}$, we obtain a field of vectors along the curve whose prolongations are tangent to another curve. (See, [5], [6]).

When a vector field $C^{h}(u)$ is given along the hypersurface $V^{n-1}$ and satisfies

$$
\nabla_{c} C^{h}=\alpha B_{c}{ }^{h}+\beta_{c} C^{h},
$$


where $\alpha$ is a scalar field and $\beta_{c}$ a covector field of the hypersurface, we say that the vector field $C^{h}(u)$ is torse-forming along the hypersurface.

In this Section, we assume that there is given a torse-forming vector field $C^{h}$ along $V^{n-1}$ which is not tangent to the hypersurface and take this vector field as the pseudo-affine normal to the hypersurface $V^{n-1}$.

We induce an affine connection on the hypersurface with respect to this torseforming vector field $C^{h}$ and call the connection of this kind the connection of Tzitzéica on the hypersurface.

When the vector field $C^{h}$ satisfies

$$
\nabla_{c} C^{h}=\alpha B_{c}{ }^{h}
$$

with a non-zero constant $\alpha$, we say that the vector field $C^{h}$ is concurrent along $V^{n-1}$ because when we develop the vector field along a curve on the hypersurface, we obtain a field of vectors along the curve whose prolongations pass through a fixed points. (See [3], [6]). In a centro-affine space with a fixed point $O$, the vector field $X=\overrightarrow{\mathrm{OP}}$ attached to a point $\mathrm{P}$ is concurrent on any hypersurface in the sense above.

When the pseudo-affine normal $C^{h}$ is torse-forming, comparing (3.5) and (4.2), we find

$$
h_{c}^{a}=-\alpha \delta_{c}^{a}
$$

and

$$
l_{c}=\beta_{c}
$$

Thus if moreover the hypersurface has the property $\left(\mathrm{P}^{\prime}\right)$, we have, from (3.9) and (3. 10),

$$
K_{d c b}{ }^{a}+\delta_{d}^{a}\left(\gamma_{c l}+\alpha h_{c b}\right)-\delta_{c}^{a}\left(\gamma_{a b}+\alpha h_{d b}\right)-\alpha_{d c} \delta_{b}^{a}=0
$$

and

$$
\nabla_{d} h_{c b}-\nabla_{c} h_{d b}+\beta_{d} h_{c b}-\beta_{c} h_{d b}=0 .
$$

Thus from Proposition 2.2 and (4.6), we see that, if $n-1 \geqq 3$, then the connection of Tzitzéica is projectively flat. Thus we have

THEOREM 4.1. The connection of Tzitzéica induced on a hypersurface $V^{n-1}$ with the property $\left(\mathrm{P}^{\prime}\right)$ in $M^{n}(n \geqq 4)$ with respect to a torse-forming pseudo-affine normal is projectively flat.

As a corollary to Theorem 4.1 , we have

Theorem 4.2. The connection of Tzitzéica induced on a hypersurface $V^{n-1}$ with the property $\left(\mathrm{P}^{\prime}\right)$ in $M^{n}(n \geqq 4)$ with respect io a concurrent pseudo-affine normal is projectively flat. 


\section{BiBLIOGRAPHY}

[1] Dobrescu, A., Sur les variétés immergées dans $E_{n_{+1}}$. Revue Roumaine de Mathématiques Pures et Appliquées 12 (1967), 829-841.

[2] Ogiue, K., On invariant immersions. To appear.

[ 3 ] Schouten, J. A., Riccı calculus. Springer-Verlag (1954).

[4] TzitzéicA, G., Oeuvres, Vol. I. Bucarest (1941).

[5] Yano, K., Sur le parallélisme et concourance dans l'espace de Riemann. Proc. Imp. Acad. Tokyo 19 (1943), 189-197.

[6] Yano, K., On the torse-forming directions in Riemannian spaces. Proc. Imp. Acad. Tokyo 20 (1944), 340-345.

Department of Mathematics,

Tokyo Institute of Technology. 